\title{
Standardised management of PID in a developing
}

\section{country}

\author{
XAVIER DE MUYLDER \\ From the Gweru Provincial Hospital, Gweru, Zimbabwe
}

SUMMARY During a 15 month period, 464 patients admitted to hospital with pelvic inflammatory disease (PID) were classified according to Monif's staging and treated following strict guidelines. Stage II, PID with peritoneal reaction, was treated with intravenous antibiotics. Stage III, PID with tubo-ovarian mass, was drained by posterior colpotomy when indicated or treated with triple antibiotics when high abdominal masses were present. Stage IV, ruptured tubo-ovarian abscess, was always surgically treated. Mortality was almost limited to patients with stage IV PID, 15\% (3/20) of whom died. Morbidity included the need for laparotomy (in $1 \cdot 6 \%(6 / 368)$ of stage II, $59 \cdot 3 \%(45 / 76)$ of stage III, and $100 \%$ of 20 stage IV patients) and draining pus (in $6.6 \%(5 / 76)$ of stage III cases and $50 \%(10 / 20)$ of stage IV patients). This study also shows that unspecialised hospital staff are able to use Monif's staging correctly, and that acceptable results can be obtained with the limited resources that are available in most developing countries.

Pelvic inflammatory disease (PID) is one of the most important consequences of sexually transmitted disease (STD) and represents the gynaecological condition afflicting African women most commonly.' In developing countries this condition constitutes a major public health challenge: high mortality, prolonged hospital stays for many patients, and multiple operations for late sequellae such as infertility, tubal pregnancy, and chronic lower abdominal pain.

PID includes a large range of clinical manifestations, but its management must be tailored to the progression of the disease. The objective classification suggested by Monif ${ }^{2}$ thus facilitates efficient clinical action. In most African hospitals, outpatient clinics are run by nurses under the supervision of a doctor, and hospital care is provided by general practitioners. This means that most patients with PID are managed by medical staff who have not specialised in STD, who should therefore be offered strict guidelines for the diagnosis, classification, and treatment of patients with PID.

The objectives of the study published here were to describe a standardised approach based upon Monif's classification, to analyse the first short term results

\footnotetext{
Address for reprints: Dr Xavier De Muylder, Department of Gynaecology, Clinique Générale Saint-Jean, 114 rue du Marais, B-100 Brussels, Belgium
}

Accepted for publication 26 February 1989 obtained, and to present the main complications observed in a Zimbabwean community during a 15 month period.

\section{Patients and methods}

I studied all women who were seen with a diagnosis of PID at this hospital from 1 January 1985 to 31 March 1986. The criteria for diagnosing PID were: lower abdominal pain of acute onset, abnormal vaginal discharge, lower abdominal rebound, cervical motion tenderness, and either fever or an erythrocyte sedimentation rate of more than $15 \mathrm{~mm}$ in the first hour. During the study period no laparoscopy was used for diagnosing or staging PID, but all patients were classified according to Monif's recommendations. In stage I, which indicates PID without peritoneal reaction and without pelvic mass, patients were treated with tetracycline by mouth for 10 days while ambulatory. In stage II, PID with peritonitis, patients were admitted to hospital for intravenous antibiotic treatment. As preliminary results had been encouraging, ${ }^{3}$ most patients received a combination of penicillin and chloramphenicol. Stage III, defined as PID with peritonitis and a pelvic mass, raised the possibility of a tubo-ovarian abscess. When there was a fluctuant mass in the pouch of Douglas, vaginal drainage was undertaken through a posterior colpotomy. When the mass was too high in the abdomen, aggressive medical treatment with penicillin, genta- 
mycin, and chloramphenicol or metronidazole was tried to avoid operating and possible salpyngooophorectomy. Finally, in the life threatening stage IV, rupture of the tubo-ovarian complex, treatment was by operating as soon as the patient's general condition was stabilised by administering antibiotics, intravenous fluids, and blood transfusion if necessary.

A patient treated medically was considered to be a treatment failure if she met any of the following three criteria: persistent fever after four days, worsening symptoms after $\mathbf{4 8}$ to $\mathbf{7 2}$ hours, or the appearance or enlargement of an adnexal mass during treatment. In these circumstances, she was given another antibiotic regimen if her PID was at stage II or surgical treatment if it was at stage III.

\section{Results}

During the study period 557 patients were admitted with a diagnosis of PID, which represented $35 \%$ of the 1592 admissions to the gynaecological ward. Of 93 who had to be excluded from the study, 48 (with ectopic pregnancy, ovarian cyst, or appendicitis) had been inaccurately diagnosed as having PID, 38 had PID without peritoneal reaction (stage I), and seven refused the suggested treatment. Table 1 shows the main morbidity features of the remaining 464 women.

Of 368 patients who presented with PID stage II, 318 were treated with penicillin and chloramphenicol. The clinical response was satisfactory in 238 (74.8\%) but this treatment failed in the other 80 patients. Except for four women who developed pelvic masses, which were surgically removed, all those in whom treatment had failed were treated with ampicillin alone or in combination with metronidazole. This second treatment was successful in 72 of the remaining 76 patients, two had to be operated on because of pelvic masses, and two responded only to triple antibiotics.

The remaining 50 patients with PID stage II received ampicillin alone (18) or in combination with chloramphenicol (10) or metronidazole (22) as first line treatment. This was successful in 46 cases, but two patients developed masses requiring surgery and two were cured only after receiving gentamycin.

Table 1 Main morbidity features according to stage of pelvic inflammatory disease (figures are numbers (percentages) of patients)

\begin{tabular}{llrrrrrrr}
\hline & $\begin{array}{l}\text { Stage II } \\
(n=368)\end{array}$ & \multicolumn{2}{c}{$\begin{array}{l}\text { Stage III } \\
(n=76)\end{array}$} & \multicolumn{2}{c}{$\begin{array}{l}\text { Stage IV } \\
(n=20)\end{array}$} & \multicolumn{2}{c}{$\begin{array}{l}\text { Total } \\
(n=464)\end{array}$} \\
\hline Mortality & 0 & & 0 & & 3 & $(15)$ & 3 & $(0 \cdot 7)$ \\
TAH + BSO* & 0 & & 4 & $(5 \cdot 3)$ & 8 & $(40)$ & 12 & $(2 \cdot 6)$ \\
Laparotomy & 6 & $(1 \cdot 6)$ & 41 & $(54 \cdot 0)$ & 12 & $(60)$ & 63 & $(13 \cdot 6)$ \\
$\begin{array}{l}\text { Colpotomy } \\
\text { Repeat surgery }\end{array}$ & 0 & $(0.5)$ & 11 & $(14 \cdot 5)$ & 0 & & 13 & $(2 \cdot 8)$ \\
$\begin{array}{l}\text { General } \\
\text { anaesthesia }\end{array}$ & 8 & $(2 \cdot 2)$ & 61 & $(8.6)$ & 10 & $(50)$ & 15 & $(3 \cdot 2)$ \\
\hline
\end{tabular}

*TAH + BSO = total abdominal hysterectomy and bilateral salpyngo-oophorectomy.
Table 2 Main complications in 20 patients with stage IV pelvic inflammatory disease

\begin{tabular}{ll}
\hline $\begin{array}{l}\text { During operation: } \\
\text { Septic shock } \\
\text { Injury to small bowel }\end{array}$ & 1 \\
Injury to rectum & 1 \\
& 1 \\
Postoperative: & \\
Pus collected again & 3 \\
Chest empyema & 2 \\
Septicaemia & 8 \\
Septic shock & 6 \\
Rectovaginal fistula & 1 \\
Wound abscess or infection & 7 \\
Pneumonia & 4 \\
Renal failure & 3 \\
Liver failure & 1 \\
\hline
\end{tabular}

Seventy six patients presented with pelvic masses complicating their PID (stage III). Four were in such a poor condition that ruptured tubo-ovarian abscesses were suspected. They were prepared for immediate surgery, but laparotomy disclosed intact pelvic abcesses. Eleven presented with a mass in the pouch of Douglas and were treated by colpotomy; 10 of them were cured, but one remained febrile and had to undergo laparotomy. In 61 women, medical treatment with penicillin, gentamycin, and chloramphenicol (or metronidazole) was tried but resulted in a cure in only 21; the other 40 were cured by surgery. No mortality occurred in stage III patients, but some postoperative complications were recorded: abdominopelvic collection of more pus (in one), subphrenic abscess (1), chest infection (1), ileus paralyticus (2), and wound abscesses (2). The mean (SD) length of hospital stay for these patients was $16.5(7 \cdot 3)$ days.

Twenty patients were admitted to the gynaecological ward with ruptured tubo-ovarian abscesses (stage IV PID), many of them having been transferred from other wards or hospitals where they had been incorrectly diagnosed and treated. At the time of laparotomy, seven had unilateral and 13 bilateral ruptured tubo-ovarian abscesses with multiple abdominal and subphrenic collections of pus. Most of these patients developed major operative and postoperative complications (table 2) requiring multiple further operations: another laparotomy (in 4), drainage of chest empyema (in 2), closure of colostomy (in 1 ), and drainage of wound abscess (in 3). Three patients died of septic shock; the mean (SD) length of hospital stay was $31.7(17 \cdot 4)$ days.

\section{Discussion}

In most developing countries, managing PID represents a real challenge to health services that are working without medical staff who specialise in STD problems, without laparoscopy to confirm the diagnosis and assess the stage of the disease, without microbiological facilities for culture and assessing sensitivity to antibiotics, and without the availability 
of a large choice of active antibiotics. In this context, efficient action implies sticking to strict guidelines for management.

Once PID has been diagnosed, the first requirement is to assess the progression of the disease. The staging suggested by Monif appears to be appropriate for this because it is based on clinical signs that can be recorded in the least sophisticated environment. After a few weeks of training, members of our hospital staff were familiar with this classification and able to use it in daily clinical practice. It is important to note that teaching had been directed mainly to taking a correct history and making a careful search for peritoneal reaction and pelvic mass.

Most patients with PID seen at the outpatient clinic were only at stage $I$. They were treated as outpatients unless the diagnosis was in doubt, in which case they were admitted to hospital until other abdominal emergencies had been ruled out. Patients with PID and signs of peritoneal reaction were always admitted to hospital for intravenous treatment. Most $\mathbf{7 9} .3 \%$, $368 / 464)$ of the women studied were at stage II, and only $16.4 \%(76)$ were at stage III and $4.3 \%(20)$ at stage IV. This distribution was very close to that found by Robson and Cloete, who reported $76.5 \%$ for stage II, $21 \cdot 2 \%$ for stage III, and $2 \cdot 3 \%$ for stage IV.

At our hospital PID stage II was often treated with a combination of penicillin and chloramphenicol, as recommended by the Zimbabwean public health authority. ${ }^{5}$ This association appeared to be successful in $75 \%$ of cases. Ampicillin, either alone or in combination with chloramphenicol or metronidazole, was mainly kept as second line treatment and was efficient in $94 \%$ of cases. Triple antibiotics were rarely needed for stage II, but during treatment eight $(2 \cdot 2 \%)$ patients developed masses that required surgical intervention.

Stage III management depends mainly on the location of the mass. When the mass could be reached through the vagina the patient was always treated by posterior colpotomy, and this simple management succeeded in 10 out of 11 patients. There are two opposing schools of thought regarding the management of abdominal masses. The first advocates early operation and removal of the mass. Kaplan et al recommended an aggressive surgical approach for all patients who exhibit no or only partial clinical response after 24-48 hours of medical management. ${ }^{6}$ The main goal of other clinicians is to avoid surgery and to preserve ovarian function by preventing the need for salpingo-oophorectomy. During the study period we favcured the second approach except in patients with suspected ruptured pelvic abscesses. Our reasons were multiple: the tubo-ovarian complex may be nothing more than adherence of omentum and oedematous bowel to the inflamed tubes, many masses resolve after antibiotic treatment only, ${ }^{7}$ and (mainly) the young ages of many patients for whom the hope of future childbearing warrants more conservative treatment whenever possible. Our present data suggest that such medical treatment was successful in only one third of the patients, the rest of the stage III patients requiring operation. This was in line with other results obtained in Zimbabwe. ${ }^{8}$ Although that result was disappointing, medical treatment did allow several patients to escape surgery, and we think that that number could increase if more sophisticated antibiotics (such as cephalosprin, clindamycin, and other aminoglycosides) were available.

This study also underlines the importance of PID, which is reflected by its incidence and its mortality and morbidity. PID accounted for $35 \%$ of all admissions to the gynaecological ward of our hospital. This is comparable with the figures reported by Lithgow and Rubin' and Brown and Cruickshank,' and emphasises the incidence of this condition in this part of the world. Mortality is still a matter of concern, with $15 \%$ of stage IV patients dying, though in our study, mortality was almost limited to that stage. Morbidity remains high, with many postoperative complications and a common need for second operations (table 2). The burden for the health system is also heavy: 3544 days in hospital and 99 general anaesthetics for 464 patients. Finally, the price paid by the women cannot be underestimated, with three maternal deaths, 12 patients undergoing radical surgery, and 75 undergoing major operations, which, together with the infection, could lead to late sequellae such as infertility, intestinal occlusion due to adhesions, and chronic lower abdominal pain.

In summary, our 15 month study confirms how serious and common PID is in developing countries. It shows that Monif's classification can be a useful tool even in the hands of unspecialised staff, and it suggests that acceptable results can be obtained with the limited resources that are available in most of these countries.

\section{References}

1 Lithgow DM, Rubin A. Pelvic inflammatory disease. In: Charlwood G, ed. Gynaecology in southern Africa. Johannesburg: Witwatersrand University Press, 1972:177-217.

2 Monif G. Clinical staging of acute bacterial salpingitis and its therapeutic ramifications. Am J Obstet Gynecol 1982;143: 489-95.

3 De Muylder X. Treatment of acute pelvic inflammatory disease with penicillin and chloramphenicol in a developing country. Ann Soc Belge Med Trop 1986;66:177-82.

4 Robson RA, Cloete AM. Pelvic inflammatory disease in Cape Town, South Africa. Journal of Obstetrics and Gynaecology 1986;6:suppl 1:S33-4.

5 Anonymous. EDLIZ (essential drugs list for Zimbabwe). Harare, Zimbabwe: Ministry of Health, 1985:29.

6 Kaplan A, Jacobs W, Ehresman J. Aggressive management of pelvic abscess. Am J Obstet Gynecol 1967;98:982-8.

7 Thompson S, Hager D, Wong J, et al. The microbiology and therapy of acute pelvic inflammatory disease in hospitalized patients. Am J Obstet Gynecol 1980;136:179-86.

8 Norman R, Brown I. Unilateral tubo-ovarian involvement in pelvic inflammatory disease. Cent Afr J Med 1980;26:213-6.

9 Brown I, Cruickshank J. Aetiological factors in pelvic inflammatory disease in urban blacks in Rhodesia. $S$ Afr Med J 1976;50:1342-4. 